\title{
SOUND RADIATED BY A WAVE-LIKE STRUCTURE IN A COMPRESSIBLE JET
}

\author{
V. V. Golubev*, A. F. Prieto ${ }^{\dagger}$, R. R. Mankbadi ${ }^{\dagger \dagger}$ \\ Embry-Riddle Aeronautical University, FL \\ M. D. Dahl ${ }^{*}$ \\ NASA Glenn Research Center, OH \\ R. Hixon \\ University of Toledo, $\mathrm{OH}$
}

\begin{abstract}
This paper extends the analysis of acoustic radiation from the source model representing spatially-growing instability waves in a round jet at high speeds. Compared to the previous work in Refs. [1-2], a modified approach to the sound source modeling is examined that employs a set of solutions to linearized Euler equations. The sound radiation is then calculated using an integral surface method.
\end{abstract}

\section{INTRODUCTION}

The current work provides the first preliminary test of a modified approach to modeling the compressible jet sound source and its acoustic radiation. The approach is considered an extension to the method originally developed in Ref. [1] and based on the integral energy approach. The method was later applied to jet noise predictions in Ref. [2].

The integral energy approach uses the assumption that the unsteady fluctuations in the noise-producing jet mixing layer are dominated by coherent, large-scale structures. The coherent structure is modeled by splitting the flow into three components, a time-average mean component, a large-scale wave-like coherent component, and a fine-scale random turbulence component. The nonlinear effects are accounted by considering interaction among various scales of motion. In particular, the kinetic energy equations (obtained from time and phase averaging of the full compressible Nervier-Stokes equations) are integrated across the jet to produce a set of ordinary differential equations describing interactions among the three flow components. Such equations are then solved to yield the development of each flow component, further used as a

\footnotetext{
*Assistant Professor, Member AIAA

†Graduate Research Assistant

$\uparrow \dagger$ Professor and Chair, Associate Fellow AIAA

$\ddagger$ Research Scientist, Member AIAA

$\ddagger \ddagger$ Associate Professor, Member AIAA
}

source for acoustic radiation predictions. In the previous work by Dahl \& Mankbadi [1], the coherent structure profiles were modeled using the locally parallel linear stability theory. The predicted shapes of the coherent structures were then used to compute the energy integrals governing the mean kinetic energy transformations and its transfer to the coherent structures. Once the energy integrals are computed, the nonlinear development of the instability wave amplitude can be determined from the solution of nonlinear differential equations.

In the present work, the linearized Euler equations are used instead of the instability wave equations to model the radial profiles. This has the advantages that: (i) it accounts for the fully non-parallel flow defects, (ii) by using a time-marching code, disturbances of multifrequency components can be directly calculated numerically rather than considering only a singlefrequency disturbance, and (iii) since the normal mode decomposition is not used in deriving the LEE equations, disturbances may be of general nature, and not necessarily the Kelvin-Helmholtz modes.

The following discussion outlines the strategy for the modified sound source modeling which is then used in the preliminary test to obtain the jet near field, followed by application of an integral surface method to predict the source acoustic radiation pattern.

\section{SOURCE MODEL FORMULATION}

A high-Reynolds number turbulent jet issuing from a nozzle of diameter $D$ in a still air is considered. The jet is assumed shock-free, and is excited by a singlefrequency instability wave of Strouhal number $S t=f D / U$. The density and the velocities are normalized by the jet exit density and velocity at the centerline.

American Institute of Aeronautics and Astronautics

This is a preprint or reprint of a paper intended for presentation at a conference. Because changes may be made before formal publication, this is made available with the understanding that it will not be cited or reproduced without the permission of the author. 
In Ref. [1], the development of large-scale, coherent, wave-like structure profiles in the compressible round jet was obtained using the integral energy method with the locally-parallel linear stability theory. The results were presented as single-frequency, single-azimuthal number modes which, for the pressure perturbation, take the form,

$$
p^{\prime}(x, r, \phi, t)=\hat{p}(r) A(x) \exp \left(i \int_{0}^{x} \alpha d \chi-i \omega t\right) \cos (n \phi)+c c,
$$

where ${ }^{\wedge}$ denotes the transversal shape function of the transversal coordinate $r$ at a given location along the jet (also, the eigenfunctions corresponding to a given $n$ and $\omega), \mathrm{n}$ is the azimuthal wave number indicating the rotation around the jet centerline, $\alpha$ is the axial wave number, $\omega$ is the excitation frequency, and $c c$ denotes the complex conjugate. $A(x)$ is the complex amplitude function of $x$ which can be determined from a nonlinear analysis once the linear evolution is obtained.

Figure 1 illustrates results obtained in Ref. [1] for the predicted development of the jet instability wave amplitude function using the linear (using locallyparallel stability theory) and nonlinear analyses, for $S t=0.2, \mathrm{n}= \pm 1$, and the jet Mach numbers $M=0.9,1.2$, 1.5, and 1.8. The Figure shows the development of the pressure wave magnitude along the nozzle lipline $(r=D / 2)$. It is observed that the nonlinear effects limit the amplitude growth and shift the peak amplitude towards the jet nozzle exit. These effects become more pronounced as the Mach number increases.
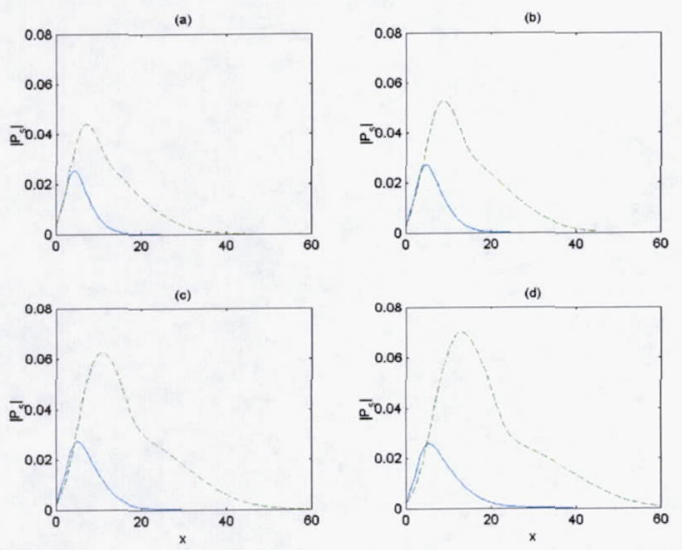

Figure 1. Axial variation of the pressure magnitude for the jet instability wave with linear (dashed line) and nonlinear (solid line) amplitude development: $r=D / 2$, St $=0.2, \mathrm{n}=1$, (a) $M=0.9$, (b) $M=1.2$, (c) $M=1.5$, (d) $M=1.8$.
In what follows, an alternative approach to calculating the linear profile of the jet instability wave is discussed.

\section{LEE APPROACH}

In the linearized Euler equations (LEE) approach (e.g., Ref. [3]), the total flowfield is split into a time-averaged mean flow and a time-dependent disturbance field, with the latter considered a small-amplitude perturbation of the mean flow. Starting with the full Navier-Stokes equations in the conservative form, neglecting viscosity, and linearizing about the mean flow Cartesian components $(U, V, W, \rho, E)$, the linearized Euler equations can be obtained in the form

$$
\frac{\partial \hat{Q}}{\partial t}+\frac{\partial \hat{F}}{\partial x}+\frac{1}{r} \frac{\partial(r \hat{G})}{\partial r}+\frac{1}{r} \frac{\partial \hat{H}}{\partial \phi}=\hat{S}
$$

where the perturbation solution vector

$$
\hat{Q}=[\hat{\rho}, \hat{u}, \hat{v}, \hat{w}, \hat{e}]^{T}
$$

is related to the primitive disturbance field variables by

$$
(\hat{u}, \hat{v}, \hat{w}, \hat{e}, \hat{\rho}, \hat{p})=\left[(\rho u)^{\prime},(\rho v)^{\prime},(\rho w)^{\prime},(\rho e)^{\prime}, \rho, p^{\prime}\right],
$$

the flux vectors are

$$
\begin{gathered}
\hat{F}=\left[\begin{array}{c}
\hat{u} \\
\hat{p}+2 \hat{u} U-\hat{\rho} U^{2} \\
\hat{u} V+\hat{v} U-\hat{\rho} U V \\
\hat{u} W+\hat{w} U-\hat{\rho} U W \\
(\hat{p}+\hat{e}) U+(\hat{u}-\hat{\rho} U) E
\end{array}\right], \\
\hat{G}=\left[\begin{array}{c}
\hat{u} V+\hat{v} U-\hat{\rho} U V \\
\hat{p}+2 \hat{v} V-\hat{\rho} V^{2} \\
\hat{v} W+\tilde{w} V-\hat{\rho} V W \\
(\hat{p}+\hat{e}) V+(\hat{v}-\hat{\rho} V) E
\end{array}\right],
\end{gathered}
$$




$$
\hat{H}=\left[\begin{array}{c}
\hat{w} \\
\hat{u} W+\hat{w} U-\hat{\rho} U W \\
\hat{v} W+\hat{w} V-\hat{\rho} V W \\
\hat{p}+2 \hat{w} W-\hat{\rho} W^{2} \\
(\hat{p}+\hat{e}) W+(\hat{w}-\hat{\rho} W) E
\end{array}\right]
$$

the non-homogeneous term is

$$
\hat{S}=\frac{1}{r}\left[\begin{array}{c}
0 \\
0 \\
\hat{p}+2 \hat{w} W-\hat{\rho} W^{2} \\
v W+w V-\rho V W \\
0
\end{array}\right],
$$

the disturbance and mean flow pressures are determined from

$$
\hat{p}=(\gamma-1)\left[\hat{e}-(\hat{u} U+\hat{v} V+\hat{w} W)+\frac{1}{2} \hat{\rho}\left(U^{2}+V^{2}+W^{2}\right)\right]
$$

and

$$
P=(\gamma-1) \rho\left[E-\frac{1}{2}\left(U^{2}+V^{2}+W^{2}\right)\right],
$$

respectively.

With no particular assumptions made regarding the form of the disturbance, LEE can be used for predicting both the flow and the acoustic disturbances. Importantly, no boundary layer type approximation is imposed on the LEE, and therefore, it can be used for predicting the unsteady flowfield of nonparallel mean flows. However, nonlinear effects are completely absent in LEE, and the mean flow is assumed to be given by some other means. In application to the jet instability wave linear analysis, the jet mean flow data is obtained from a Parabolic Navier-Stokes (PNS) solver by Dahl [4].

The current version of the LEE code [5] uses a MacCormack-type solver that is formally $4^{\text {th }}$ order accurate both in time and space. Its derivation employed Tam and Webb's DRP methodology [6].

Boundary condition treatment is the crucial step in the LEE solver implementation, developed to avoid any non-physical oscillations in the domain. The employed grid layout is depicted in Figure 2. At the inflow (left) boundary, two conditions are implemented. The first, applied to hydrodynamic flow disturbances at $\mathrm{r} / \mathrm{D}<2$, is the Thompson boundary condition [7]; the second, applied for $r / D>2$, is the conventional acoustic radiation boundary condition, also implemented at the upper boundary of the domain. At the outflow (right) boundary, Tam and Webb's [6] condition is applied. The LEE code is written in a quasi-curvilinear form, allowing stretching both in radial and axial directions. All axial stations have the same radial point distribution and vice versa (in Figure 2). Thus, the nozzle exit plane at the inflow boundary effectively determines the global grid layout. More details on the numerical implementation can be found in Ref. [5].

The test calculation was performed for a cold jet with exit Mach number $M=1.8$, excited with a helical mode $\mathrm{n}= \pm 1$ at a single frequency corresponding to Strouhal number $S t=0.2$. The PNS solver results were interpolated (and extrapolated) to the stretched LEE grid with dimensions $\left(\mathrm{N}_{\mathrm{x}} * \mathrm{~N}_{\mathrm{r}}\right)=(251 * 171)$ over the domain $\left(\mathrm{L}_{\mathrm{x}} * \mathrm{~L}_{\mathrm{r}}\right)=(60 * 35)$ (all lengths here and everywhere are normalized by $D / 2$ ). In particular, the mean flow was uniformly extrapolated in the radial direction beyond the PNS solver computational domain to the upper LEE solver boundary. The LEE code was run with global time stepping corresponding to $\mathrm{CFL}=1.4$, until convergence criteria were satisfied.

Figure 3 presents a shaded contour plot for the imported mean flow variable $\rho U$ over the LEE computational domain. Figure 4 provides illustration for the instantaneous unsteady pressure contours obtained from the LEE solver in the test run. The latter result representing obtained from LEE acoustic radiation pattern will be re-examined below in comparison with another sound prediction technique. In the jet near field, an FFT of the unsteady flowfield solution produced a linear profile of the instability mode as shown in Figure 5 for the real part of the unsteady pressure wave along the nozzle lipline.

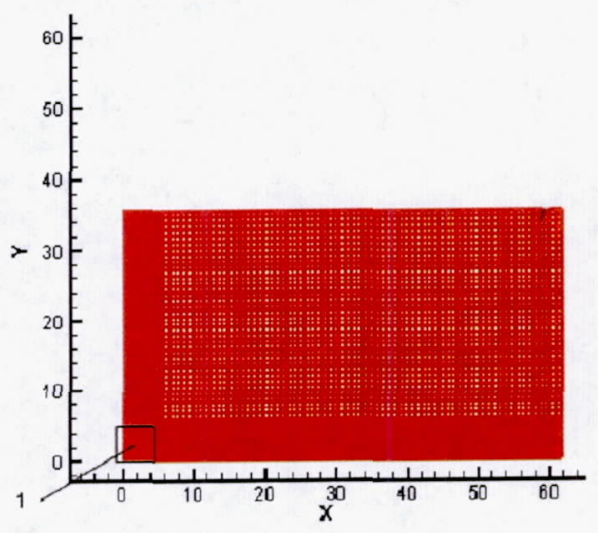

Figure 2. Grid over LEE computational domain. 


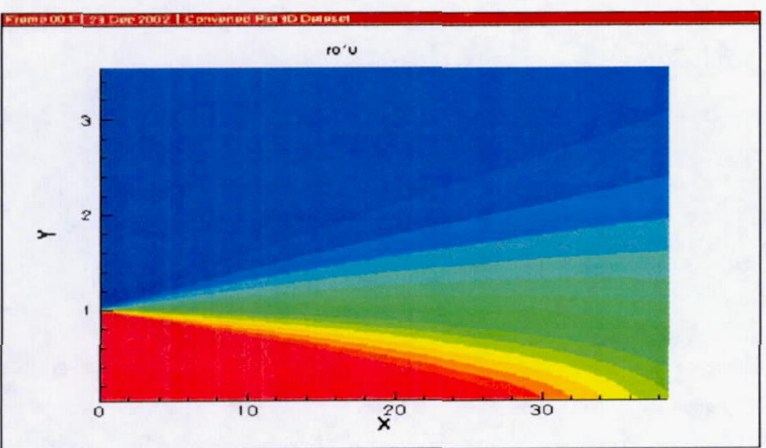

Figure 3. Mean flow ( $\rho U$ variable shown) imported in the LEE computations from the PNS solver [4].

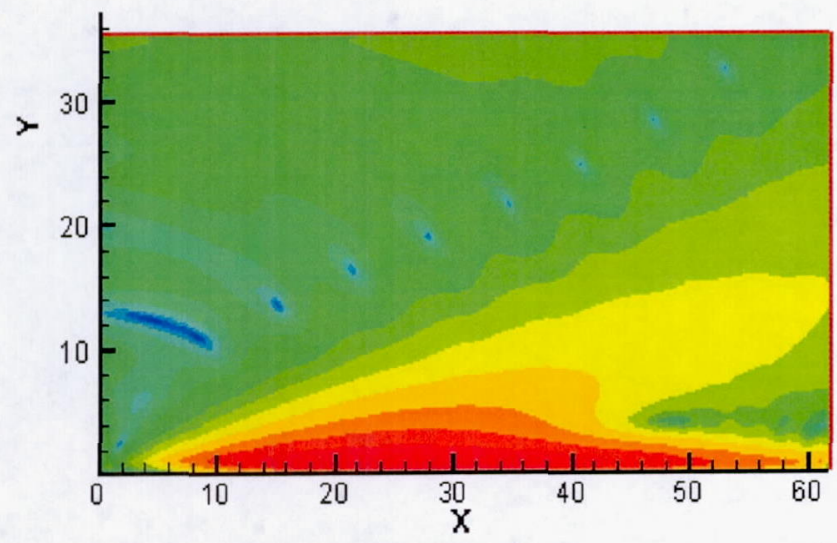

Figure 4. LEE solution for the instantaneous unsteady pressure contours for the test case.

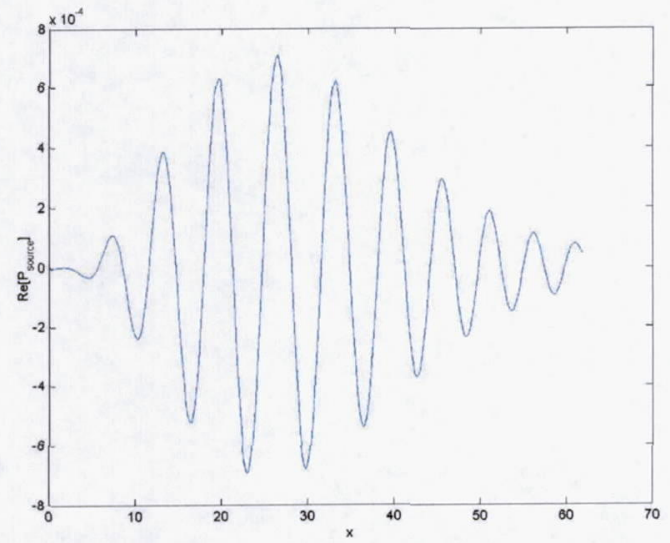

Figure 5. LEE solution for the jet instability wave acoustic source $(\operatorname{Re}[\mathrm{p}]$ is shown at $r=D / 2)$.

\section{NONLINEAR SOURCE CORRECTION}

Once the linear profile of the instability mode is obtained from the LEE solver, a nonlinear analysis of the complex amplitude function in Eq. (1) can be conducted through construction of the energy integrals and solutions to a nonlinear set of ordinary differential equations as indicated in Ref. [1]. A detailed analysis of this step is the subject of the future work. Here, just to illustrate the concept for our test case above, an assumption will be employed that the nonlinear correction function in this case would approximately correspond to the ratio of the linear and nonlinear amplitudes of the sound source obtained in Ref. [1] and presented in Figure 1(d), with the same drop in maximum (saturation) amplitude. This leads to the variation of the pressure magnitude for the source as illustrated in Figure 6.

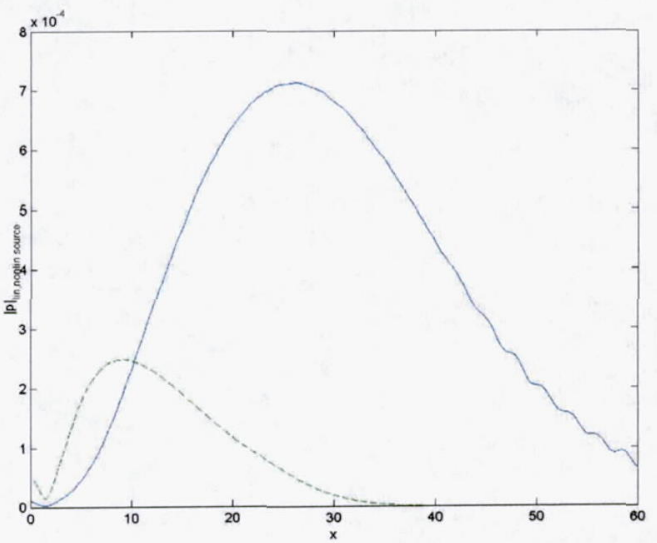

Figure 6. Pressure magnitude for LEE acoustic source (solid line) and its (assumed for the test) nonlinear correction (dashed line) along the Kirchhoff surface.

\section{FORMULATION FOR SOUND PREDICTION}

By substituting the nonlinear amplitude function obtained above into Eq. (1), the formulation for the sound source of a jet excited with a single-frequency, single-azimuthal number helical mode is completed. The far field acoustic radiation from this source is obtained using a Surface-Integral Formulation (SIF) [8]. This method is based on evaluating the near-field unsteady flow data on a control surface surrounding the nonlinear flow region, and propagating the unsteady flow information to the far field through the linear flow 
region. The method belongs to a family of Kirchhoffsurface methods [9] but is modified from the classical formulation in order to eliminate the need to estimate pressure derivatives on the control surface. In application to the present study, a control surface is considered as a cylinder of radius $a$ and length $L$ enclosing all the jet acoustic sources, as illustrated in Figure 7. It will be further assumed that the mean flow outside the cylinder is stagnant, and thus the acoustic disturbances are described by the simple wave equation in the cylindrical coordinates:

$$
\nabla^{2} p-\frac{1}{c^{2}} \frac{\partial^{2} p}{\partial t^{2}}=0
$$

Since the jet pressure modes (1) are excited with a specified frequency $\omega$, it is convenient to transform the formulation to the frequency domain by applying the Fourier transform to (3). The integral solution is then obtained in the form,

$$
p(\mathbf{X}, \omega)=-\int\left[G \frac{\partial p}{\partial n}-p \frac{\partial G}{\partial n}\right] d S
$$

where $p(\mathbf{X}, \omega)$ is the acoustic pressure at the observation point $\mathbf{X}=(X, R, \Phi), G$ is the Green function, $\mathbf{n}$ is the normal to the surface $S$, and $p(\mathbf{x}, \omega)$ is the pressure distribution on the control surface at a point $\mathbf{x}=(x, r, \phi)$.

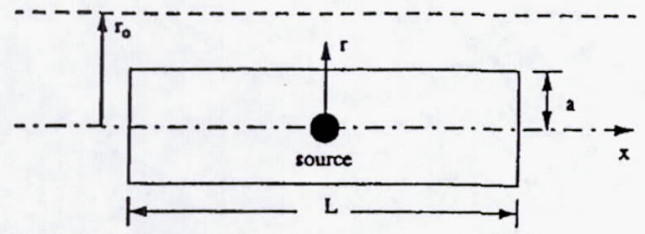

Figure 7. Cylinder used for validation of numerical codes.

The approach of the SIF formulation is to seek a solution such that $G=0$ on the control surface $r=a$, using the method of images. Morse and Ingard [10] give the Green function for emission from a cylinder of radius $a$ at a point $R$, for $R>a$, as

$$
\mathrm{g}_{1}=\Xi \int_{-\infty}^{\infty} e^{i k_{x}(X-x)} J_{m}(q r) H_{m}(q R) d k_{x}
$$

where

$$
\Xi=\frac{i}{8 \pi} \sum_{m=0}^{m=\infty} \varepsilon_{m} \cos [m(\phi-\Phi)]
$$

and

$$
\begin{array}{ll}
q=\sqrt{k^{2}-k_{x}^{2}} & \text { for } k_{x}<k \\
q=i \sqrt{k_{x}^{2}-k^{2}} & \text { for } k_{x}>k,
\end{array}
$$

where $\varepsilon_{m}$ is Neumann constant, $\varepsilon_{m}=1$ for $m=0$, and $\varepsilon_{m}$ $=2$ for $m>0$. $J_{m}$ is the $m$ th order Bessel function, $H_{m}$ is the $m$ th order Hankel function of the first kind, and $k_{x}$ is the wavenumber in the $x$-direction of the acoustic disturbance at the surface. For the image of the point $R$ at $a^{2} / R$, the Green function is

$$
\mathrm{g}_{2}=\Xi \int_{-\infty}^{\infty} e^{i k_{x}(X-x)} H_{m}(q r) J_{m}\left(q \frac{a^{2}}{R}\right) d k_{x}
$$

Adding the two solutions (5) and (6), one obtains, after algebraic manipulations, the required Green function which satisfies the control surface condition $G(r=a)=$ 0 ,

$$
\begin{aligned}
G=\Xi & \int_{-\infty}^{\infty} e^{i k_{x}(X-x)} \frac{H_{m}(q R)}{H_{m}(q a)} \times \\
& {\left[J_{m}(q r) H_{m}(q a)-J_{m}(q a) H_{m}(q r)\right] d k_{x} }
\end{aligned}
$$

and, using at $r=a$ the relationship

$$
H^{\prime}(u) J(u)-J^{\prime}(u) H(u)=\frac{2 i}{\pi u},
$$

one also obtains,

$$
\begin{array}{r}
\frac{\partial G}{\partial r}=\frac{1}{4 \pi^{2} a} \sum_{m=0}^{m=\infty} \varepsilon_{m} \cos [m(\phi-\Phi)] \times \\
\int_{-\infty}^{\infty} e^{i k_{x}\left(x_{o}-x\right)} \frac{H_{m}(q R)}{H_{m}(q a)} d k_{x}
\end{array}
$$

With $G=0$ at the control surface, the integral solution reduces to

$$
p(X, R, \Phi)=\int p \frac{\partial G}{\partial r} a d x d \phi
$$


Substituting, the acoustic field is obtained as

$$
\begin{aligned}
& p(X, R, \Phi)=\frac{1}{4 \pi^{2}} \iint p(x, a, \phi) \sum_{m=0}^{m=\infty} \varepsilon_{m} \cos [m(\phi-\Phi)] \\
& \times \int_{-\infty}^{\infty} e^{i k_{x}(X-x)} \frac{H_{m}(q R)}{H_{m}(q a)} d k_{x} d x d \phi
\end{aligned}
$$

The formula (10) describes the relation between the acoustic far-field and the pressure distribution on a cylindrical control surface surrounding the jet noise sources. To perform the volume integration, the surface pressure on the cylindrical surface is taken as

$$
p(x, a, \phi, \omega)=\sum_{n=0}^{n=\infty} p_{n}(x, a, \omega) \cos n \phi
$$

and the integration over the azimuthal direction is then performed to obtain the final formula,

$$
\begin{gathered}
p(X, R, \Phi, \omega)= \\
\frac{1}{2 \pi} \sum_{m=0}^{m=\infty} \cos m \Phi \int_{0}^{L} p_{m}(x, a, \omega) \times \\
\int_{-k_{L}}^{k_{L}} e^{i k_{x}(X-x)} \frac{H_{m}(q R)}{H_{m}(q a)} d k_{x} d x
\end{gathered}
$$

where $k_{L}$ is selected as the Nyquist limit, $k_{L}=\pi / \Delta x$, and $\Delta x$ is the spacing between the $x$-points on the cylinder. The order of integration in (12) can be reversed. In Ref. [2], the SIF formulation was numerically validated against the point source test and used for jet noise predictions. For the numerical implementation, the frequency-domain analysis is carried out by first factoring out time $t$ in (1). The contribution from the cylinder bases is not taken into account (justified if the source decays enough before it reaches the base boundary), and the ratio $L / a$ should be large enough for accurate predictions. In all the computations, the number of points per wavelength in each direction is at least 12 , thus providing an error of less then $0.5 \%$ according to estimates in [9]. The code is very fast and requires only seconds of CPU time per observer point on the PC computer.

\section{TEST CASE RESULTS}

The SIF formulation is applied here to predict the acoustic radiation pattern produced by the coherent cold jet structure described by (1). As in the LEE test predictions above, a helical mode with $n= \pm 1$ is excited with Strouhal number $S t=0.2$, and the acoustic response is calculated for the jet Mach number $M=1.8$.

In the SIF computations, the right boundary of the control surface should be taken far enough so that the sound source has practically decayed and no contribution from the base is expected. Since the original source data for the linear amplitude development is produced from LEE calculations, the right base boundary is coincident with the right outflow boundary in the LEE computations. In the SIF code, the source data is read at uniformly discretized axial locations, and produced by interpolating from a nonuniform mesh used for the linear source predictions in the LEE code. A sensitivity study for the placement of the control surface revealed that the proper control surface radius was stable in all test calculations and stayed in the range of $r_{\mathcal{S}} /(D / 2)=1.5 \ldots 2.0$. Below this limit, the source is not adequately enclosed by the control surface; and for larger values of $r_{s}$, the source has sufficiently decayed so the data input is not adequate for accurate acoustic predictions. In that case, as $r_{s}$ further increases, the results usually show decreasing values of the acoustic pressure amplitude at a fixed far-field observer location.

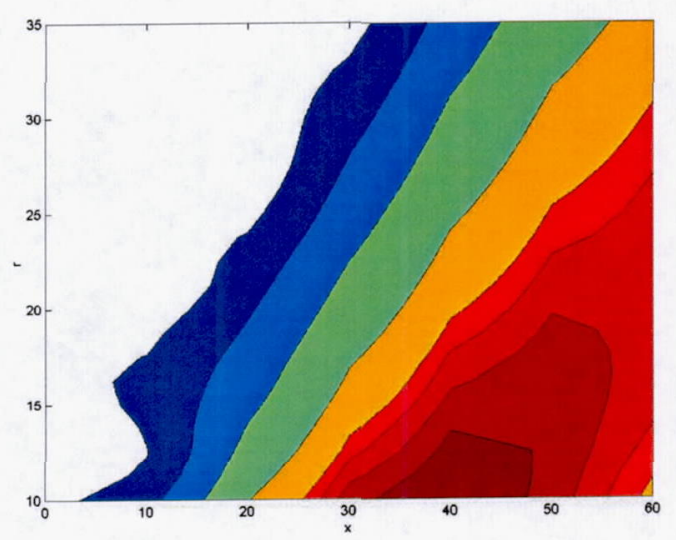

Figure 8. SPL contours for linear source amplitude.

Figures 8 and 9 illustrate the sound pressure level (SPL) contours calculated for the linear and nonlinear acoustic source wave amplitude development, respectively. As expected, numerical results showed that the acoustic response was much higher in the linear case.

The linear source acoustic predictions should also be comparable with direct far-field LEE calculations. 


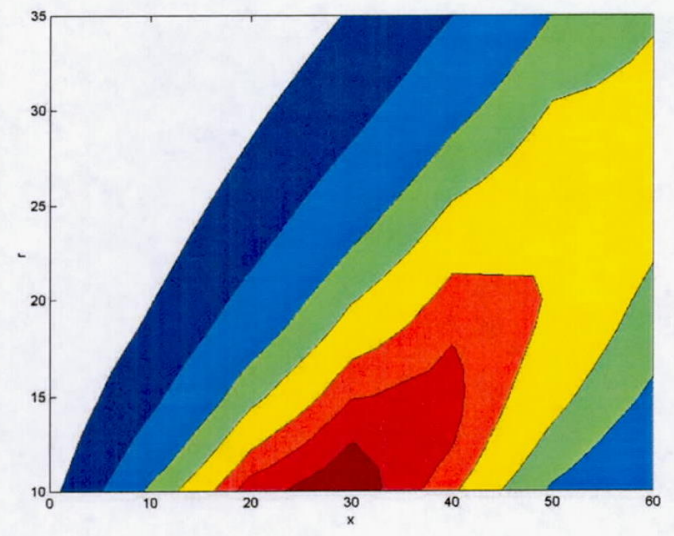

Figure 9. SPL contours for nonlinear source amplitude.

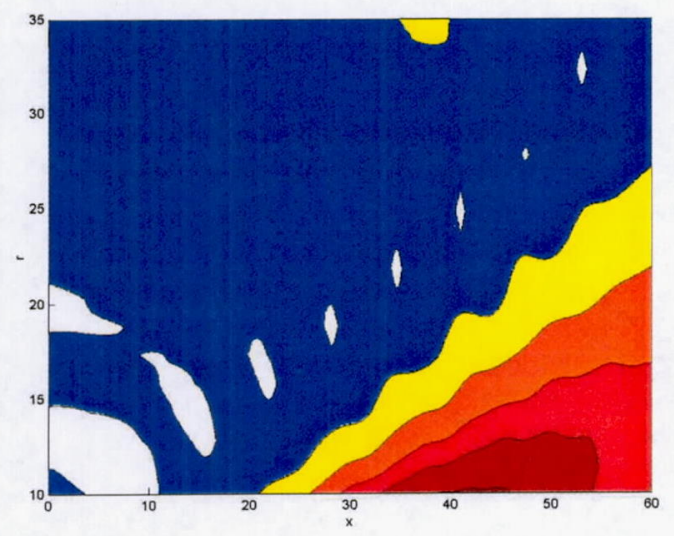

Figure 10. SPL contours from LEE predictions.

However, although sound directivities are close in both, the SPL contours from the LEE predictions illustrated in Figure 10 (and corresponding to results in Figure 4) indicated different magnitudes of sound intensity compared to SIF predictions, with difference increasing at larger distances from the jet centerline (hence, LEE predictions show faster decay). This may indicate that, although accurate enough for sound source near-field, the LEE solution has not yet been completely established (converged) throughout the domain. The differences will be further investigated in the future work.

\section{SUMMARY}

A first test of a modified approach to modeling the compressible jet sound source and its acoustic radiation was presented. The approach is viewed as an extension of the method originally developed in Ref. [1] and based on the integral energy approach. In contrast, the linearized Euler equations were used instead of the instability wave equations to model the radial profiles. In general, this approach has the advantages that: $(i)$ it accounts for the fully non-parallel flow defects, (ii) by using a time-marching code, disturbances of multifrequency components can be directly calculated numerically rather than considering only a singlefrequency disturbance, and (iii) since the normal mode decomposition is not used in deriving the LEE equations, disturbances considered can be of general nature, and not necessarily the Kelvin-Helmholtz modes. Steps in the source modeling procedure were discussed and illustrated for a test case by applying an integral surface method to predict the source acoustic radiation pattern.

\section{ACKNOWLEDGEMENTS}

Dr. Golubev would like to acknowledge the support from the ERAU Research and Professional Development Funds in this work.

\section{REFERENCES}

1. Dahl, M.D. and Mankbadi, R.R., "Analysis of ThreeDimensional, Nonlinear Development of Wave-Like Structure in a Compressible Jet," AIAA 2002-2451, 2002.

2. Golubev, V.V., Mankbadi, R.R., and Dahl, M.D., "Prediction of the Acoustic Field Associated with Instability Wave Source Model for a Compressible Jet," AIAA 20022455, 2002.

3. Mankbadi, R.R., Hixon, D.R., Shih, S.-H., and Povinelli, L.A., "Use of Linearized Euler Equations for Supersonic Jet Noise Prediction,” AIAA J., Vol. 36, pp. 140-147, 1998.

4. Dahl, M.D., "The Aeroacoustics of Supersonic Coaxial Jets," NASA TM 106782, 1994.

5. Hixon, R., Shih, S.-H., and Mankbadi, R.R., "Effect of Coannular Flow on Linearized Euler Equation Predictions of Jet Noise,", NASA CR 202339, 1997.

6. Tam, C.K.W., and Webb, J.C., "Dispersion-RelationPreserving Finite Difference Schemes for Computational Acoustics,” J. Comp. Physics, Vol. 107, pp. 262-281, 1993.

7. Thompson, K.W., "Time-Dependent Boundary Conditions for Hyperbolic Systems II,” J. Comp. Physics, Vol.89, pp.439-461, 1990.

8. Mankbadi, R.R., Shih, S.-H., Hixon, D.R., Stuart, J.T. and Povinelli, L.A., "A Surface-Integral Formulation for Jet Noise Prediction Based on the Pressure Signal Alone," J. Comp. Acoustics, Vol. 6, pp. 307-320, 1998.

9. Lyrintzis, A.S., "The Use of Kirchhoff's Method in Computational Aeroacoustics," ASME J. Fluids Eng, Vol.116, pp.665-676, 1994.

10. Morse, P.M. and Ingard, K.U., "Theoretical Acoustics," Princeton Univ. Press, 1986. 